\title{
Desempeño docente en la formación de estudiantes de Bioquímica y Farmacia, Universidad Técnica de Machala
}

\section{Teaching performance in the training of students of Biochemistry and Pharmacy, Universidad Tecnica de Machala}

Liliana Alexandra Cortez Suárez Universidad Técnica de Machala (Ecuador) lcortez@utmachala.edu.ec

Revista Cumbres Vol.4 №2

Versión impresa ISSN 1390-9541

Versión electrónica ISSN 1390-3365

http://investigacion.utmachala.edu.ec/revistas/index.php/Cumbres 


\title{
RESUMEN
}

La educación superior en el Ecuador está presentando cambios profundos orientados a la calidad y mejoramiento de la gestión académica. Este camino hacia la excelencia empieza con una adecuada evaluación de la práctica docente. Por tanto, el objetivo de la investigación fue evaluar el desempeño docente, además de valorar la percepción de los estudiantes de la Carrera de Bioquímica y Farmacia de la Universidad Técnica de Machala acerca de la calidad en su formación profesional, permitiendo además conocer el grado de incidencia entre estos dos factores. Los resultados de las encuestas realizadas a los estudiantes y profesores, revelaron valoraciones muy positivas tanto en el desempeño docente como en la calidad en la formación profesional de los estudiantes, además de una correlación positiva moderada fuerte entre estas dos variables.

Palabras clave: educación superior, evaluación, desempeño docente, formación profesional, estudiantes.

\begin{abstract}
Higher education in Ecuador is presenting profound changes oriented to the quality and improvement of academic management. This path to excellence begins with an adequate evaluation of the teaching practice. Therefore, the objective of the research was to evaluate the teaching performance, in addition to assessing the perception of the students of the Career of Biochemistry and Pharmacy of the Universidad Técnica de Machala about quality in their professional training, also allowing to know the degree of incidence between these two factors. The results of the surveys made to the students and professors, revealed very positive evaluations both in the teaching performance and in the quality in the professional formation of the students, in addition to a strong moderate positive correlation between these two variables.
\end{abstract}

Key words: higher education, evaluation, teacher performance, professional training, students. 


\section{INTRODUCCIÓN}

La actividad docente ha de estar en una continua búsqueda de mejoramiento, más aún si esta se circunscribe en los niveles de enseñanza superior. "A la docencia universitaria se le atribuye una gran responsabilidad, debido a su influencia decisiva en la formación de profesionales que ingresaran al mundo laboral y social” (Monereo \& Dominguez, 2014), por tanto, su misión es proporcionar a los estudiantes "las herramientas fundamentales para cimentar sus conocimientos ajustándolos a su realidad, permitiendo la transformación de la sociedad mediante la generación de conocimientos" (Ramírez, 2017).

En este contexto se hace necesaria la implementación de procesos de evaluación dirigidos a medir, valorar y controlar los resultados de la gestión de educación superior, a través de un análisis del desempeño de los docentes. Dimaté, Tapiero, González, Rodríguez y Arcila (2017) indican que la evaluación es un proceso continuo y sistemático que recoge datos cuantitativos y cualitativos, encaminado a la toma de decisiones, fundamentado en criterios prestablecidos y orientado a la transformación y mejoramiento de la calidad de los procesos de enseñanza-aprendizaje.

En el Ecuador, desde el 2010 se emprendió el proyecto de nuevo modelo de gestión educativa, donde uno de sus ejes "es que los docentes universitarios se conviertan en profesionales que impulsen el desarrollo venidero del país" (Ronquillo, Moreira, \& Verdesoto, 2016). El Sistema Nacional de Información de la Educación Superior del Ecuador (SNIESE), reveló los siguientes datos:

En las universidades públicas, el porcentaje de docentes con título de tercer nivel asciende al 38\%; en las cofinanciadas, el $43 \%$ y en las particulares autofinanciadas, el $42 \%$. Respecto a docentes con maestría, en las instituciones de educación superior públicas representan el 39\%; las cofinanciadas, el 28\%; y en las autofinanciadas, el 35\%. En cuanto a docentes con grado de doctorado o PhD son muy pocos. (Secretaría Nacional de Planificación y Desarrollo, 2013)

En el ámbito normativo, en octubre de 2010, se puso en marcha la Ley Orgánica de Educación Superior (LOES) y su Reglamento, teniendo por misión fortalecer el sistema universitario ecuatoriano, para que esta contribuya en transformación de la sociedad, a través de la formación de profesionales altamente capacitados, con conocimientos científicos que respondan a las necesidades del desarrollo nacional y a la construcción de ciudadanía.

De esta forma, "la educación superior demandó la profesionalización del cuerpo docente universitario para realzar la calidad educativa, a fin de fortalecer el proceso enseñanza-aprendizaje" (Moreira, 2015). En consecuencia, el desempeño docente viene siendo parte importante en los procesos educativos. Las nuevas reformas educativas del país dieron paso a la revisión consciente y dinámica de nuevas alternativas que se puedan aplicar para el desarrollo y mejoramiento de la enseñanza en todas sus manifestaciones.

En concordancia con los procesos de mejoramiento del sistema universitario ecuatoriano, y de acuerdo al art. 155 de la LOES, los profesores deben evaluados periódicamente en su desempeño académico. La evaluación del desempeño docente en el ámbito de la educación superior debe estar enfoca- 
do en la medición de la productividad investigativa y la prestación de servicios, pero principalmente a la actividad de enseñanza, a fin de proporcionar estándares de calidad que eleven el nivel de aprendizaje de los estudiantes considerando las nuevas tendencias en la educación superior. De acuerdo a Cuevas (2015) la evaluación del desempeño docente permite mejorar tanto sus prácticas como el rendimiento de los estudiantes..., considera que es fundamental para tener parámetros comparativos entre lo ideal y lo real.

Considerando estos argumentos, la presente investigación se desarrolló en la Universidad Técnica de Machala, la misma que durante el año 2012, de acuerdo a los informes del Consejo de Evaluación, Acreditación y Aseguramiento de la Calidad de la Educación Superior (CEAACES), se ubicó en la categoría D dentro del sistema de educación superior del país. Luego de tres años de gestión y la implementación de un plan de trabajo, que consistió en la inversión de infraestructura y el fortalecimiento en el área de investigación, se pudo lograr el objetivo de sacar a la universidad del sitio que se encontraba ubicada. Es así que en el año 2016, la universidad consiguió la acreditación, catalogándola como un centro de educación superior de categoría B.

Con el fin de seguir contribuyendo con el fortalecimiento de la Universidad Técnica de Machala, y de forma específica en lo relacionado al talento humano (docentes) de la carrera de Bioquímica y Farmacia, se ha seleccionado esta temática sobre la evaluación del desempeño docente como parte de un proceso que promueva una nueva cultura de la calidad, a partir de una reflexión compartida entre los diferentes actores que participan en este proceso, para posibilitar espacios abiertos para el desarrollo profesional del educador.

El objetivo de la investigación fue evaluar el desempeño docente, además de valorar la percepción de los estudiantes de la Carrera de Bioquímica y Farmacia de la Universidad Técnica de Machala acerca de la calidad en su formación profesional, así como averiguar el grado de incidencia entre estos dos factores, para conocer a profundidad los procesos y resultados de enseñanza, y de esta manera contribuir con mecanismos de control sobre el accionar de los docentes para su mejoramiento continuo.

\section{MATERIALES Y MÉTODOS}

Tipo de la investigación: es de tipo básico, ya que consistió en la búsqueda de un conocimiento puro a través de la recolección de datos, para de esta forma profundizar aún más en las teorías educativas ya existentes.

Diseño de la investigación: es correlacional, con los datos obtenidos se espera averiguar el grado de incidencia que tiene el desempeño docente sobre la calidad en la formación profesional de los estudiantes.

Procesamiento de los datos: se utilizó el software IBM SPSS Statistics versión 24, el cual permitió la tabulación, cálculos de correlación, así como la construcción de tablas y gráficos estadísticos, que ayudaron al análisis de los datos. De igual manera, se empleó hojas de cálculo de Excel. 
Población: se seleccionó a los estudiantes de la carrera de Bioquímica y Farmacia de la Universidad Técnica de Machala, que comprende una cantidad de 44 personas. Asimismo, se escogió a 23 docentes.

Técnicas de investigación: la encuesta.

Instrumentos de recolección de datos: se diseñó tres cuestionarios:

Cuestionario de evaluación del desempeño docente. Dirigido a los estudiantes, contiene 42 ítems, y valora cuatro dimensiones sobre el desempeño docente: Planificación docente, desarrollo de la enseñanza, evaluación y actitud del docente.

Cuestionario de evaluación de la calidad profesional de los estudiantes. Dirigido a los estudiantes, se compone de 30 ítems que valoran las siguientes dimensiones: Programa de estudio, medios para la formación profesional, resultados.

Cuestionario de autoevaluación del desempeño docente. Aplicado a los docentes, consta de 42 ítems. Analiza las mismas dimensiones del primer cuestionario.

Los cuestionarios utilizan la escala de Likert valorando en función al «grado de acuerdo o desacuerdo» de las declaraciones formuladas en cada dimensión. Para la interpretación de la información obtenida en los cuestionarios, se empleó una baremación o escala de medición de cinco niveles como se indica en la tabla 1:

\begin{tabular}{|c|c|c|c|c|c|c|}
\hline \multirow[t]{2}{*}{ CUESTIONARIOS } & \multirow{2}{*}{$\begin{array}{l}\text { \# } \\
\text { İTEMS }\end{array}$} & \multicolumn{5}{|l|}{ ESCALA } \\
\hline & & $\begin{array}{l}\mathrm{M} \mathrm{u} y \\
\text { bajo }\end{array}$ & Bajo & Moderado & Alto & Muy alto \\
\hline Desempeño docente & 42 & $42-67$ & $68-92$ & $93-117$ & $118-142$ & $143-168$ \\
\hline $\begin{array}{l}\text { Calidad profesional de es- } \\
\text { tudiantes }\end{array}$ & 30 & $30-48$ & $49-66$ & $67-84$ & 85-102 & $103-120$ \\
\hline $\begin{array}{l}\text { Autoevaluación desempeño } \\
\text { docente }\end{array}$ & 42 & $42-67$ & $68-92$ & $93-117$ & $118-142$ & $143-168$ \\
\hline
\end{tabular}

Hipótesis: Existe una incidencia importante entre el desempeño docente y la calidad de la formación profesional de los estudiantes de la Carrera de Bioquímica y Farmacia de la Universidad Técnica de Machala.

Variables: variable 1: Desempeño docente; y, variable 2: Calidad de la formación profesional de los estudiantes; $y$, sus dimensiones e indicadores que permitieron el desarrollo de la investigación se detallan en la Tabla 2. 


\begin{tabular}{|c|c|c|}
\hline VARIABLES & DIMENSIONES & INDICADORES \\
\hline \multirow[t]{4}{*}{$\begin{array}{l}\text { Evaluación del desempe- } \\
\text { ño docente }\end{array}$} & Planificación docente & $\begin{array}{l}\text {-Información sobre la asignatura } \\
\text {-Preparación y organización de actividades de } \\
\text { la asignatura }\end{array}$ \\
\hline & $\begin{array}{l}\text {-Desarrollo de la ense- } \\
\text { ñanza }\end{array}$ & $\begin{array}{l}\text {-Cumplimiento de obligaciones } \\
\text {-Coordinación del plan de enseñanza } \\
\text {-Metodología } \\
\text {-Conocimiento de la materia }\end{array}$ \\
\hline & -Evaluación & $\begin{array}{l}\text {-Mecanismos de evaluación } \\
\text {-Calificación de evaluaciones }\end{array}$ \\
\hline & -Actitud del docente & $\begin{array}{l}\text {-Resolución de problemas } \\
\text {-Trato con estudiantes } \\
\text {-Orientación hacia el estudiante }\end{array}$ \\
\hline \multirow[t]{3}{*}{$\begin{array}{l}\text { Evaluación calidad de la } \\
\text { formación profesional }\end{array}$} & -Programa de estudio & $\begin{array}{l}\text {-Contenidos } \\
\text {-Cumplimiento de contenidos } \\
\text {-Objetivos del programa de estudio }\end{array}$ \\
\hline & $\begin{array}{l}\text {-Medios para la forma- } \\
\text { ción profesional }\end{array}$ & $\begin{array}{l}\text {-Elementos académicos } \\
\text {-Materiales } \\
\text {-Prácticas }\end{array}$ \\
\hline & -Resultados & $\begin{array}{l}\text {-Satisfacción del estudiante } \\
\text {-Motivación al aprendizaje del alumno }\end{array}$ \\
\hline
\end{tabular}

Tabla 2. Variables, dimensiones e indicadores del objeto de investigación

Estrategia de comprobación de hipótesis: La prueba de hipótesis se ha realizado utilizando procedimientos de estadística no paramétricas, mediante el cálculo del coeficiente de correlación de Spearman, $\rho$ (rho), el mismo que mide la relación o interdependencia existente entre dos las variables que compone la hipótesis.

\section{RESULTADOS Y DISCUSIÓN}

En la encuesta de evaluación del desempeño docente aplicada a los estudiantes de la Carrera de Bioquímica y Farmacia de la Universidad Técnica de Machala durante el año 2018, se revelan en la Figura 1, en la cual de forma general los encuestados proporcionan una valorización muy alta y alta del $45,45 \%$ respectivamente, lo que indica una calificación altamente positiva por parte de los alumnos sobre la gestión desarrollada por los catedráticos. El 6,82\% manifestó que el desempeño docente es moderado; y, el 2,27\% opina que muy bajo.

En la Figura 2, se analiza por dimensiones la evaluación del desempeño docente, así tenemos, que, respecto a la planificación de las actividades docentes, el 45,45\% de los estudiantes dan una calificación alta; el 43,18\% la ubica en un nivel muy alto. En relación al desarrollo de la enseñanza, los alumnos indican un nivel de satisfacción muy alta y alta del 43,18\% para cada respuesta. El $45,45 \%$ de los estudiantes ubica en un nivel muy alto las actividades de evaluación llevadas a cabo por el docente; el 43,18\% considera que las evaluaciones tienen un nivel alto. La actitud del docente tiene una valorización muy alta en un $47,73 \%$ y alta en un 43,18\%. En cada una de las dimensiones evaluadas, los estudiantes proporciones calificaciones muy positivas a la gestión educadora de los docentes de la Carrera de Bioquímica y Farmacia de la Universidad Técnica de Machala. 


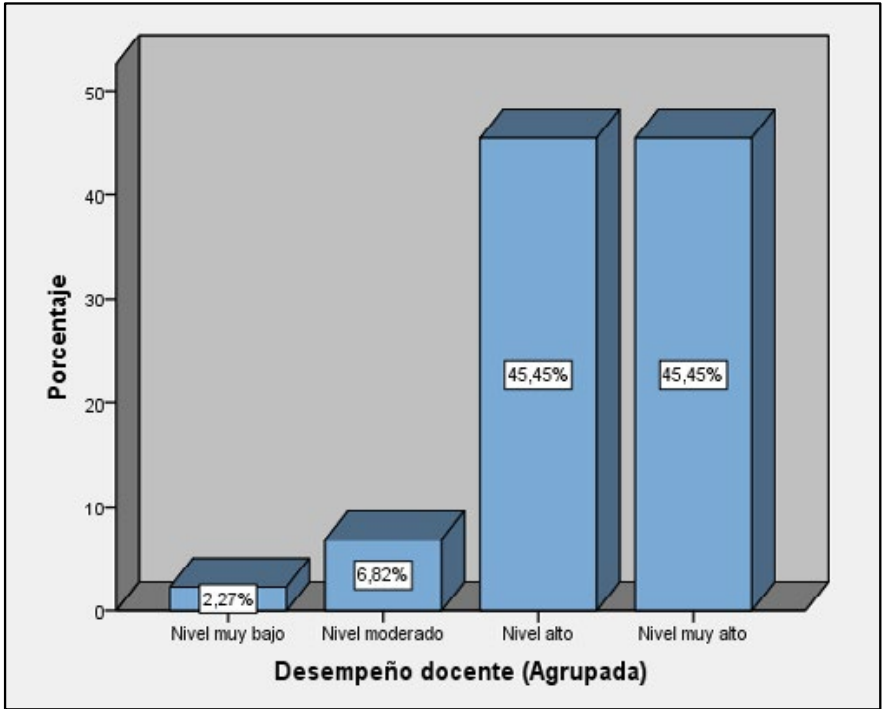

Figura 1. Evaluación del desempeño docente

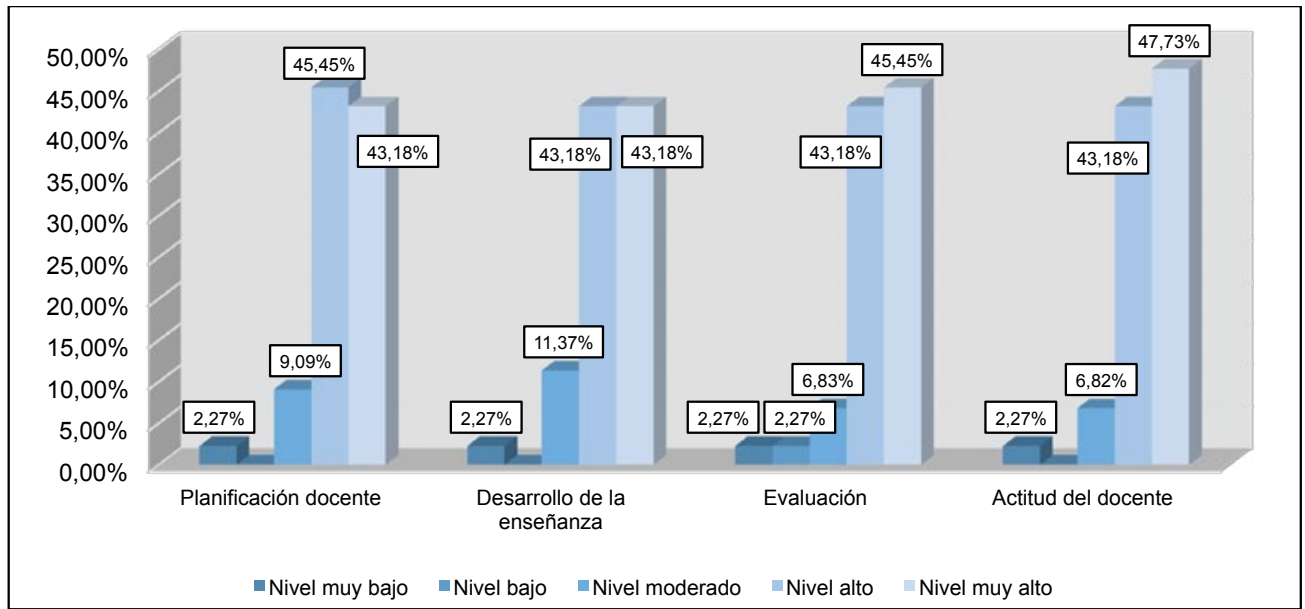

Figura 1. Resultados de la evaluación del desempeño docente por dimensiones

Continuando con el estudio, la segunda encuesta dirigida a los estudiantes de la Carrera de Bioquímica y Farmacia de la Universidad Técnica de Machala, tuvo por finalidad conocer su opinión acerca si las actividades docentes están contribuyendo en su formación profesional con estándares de calidad.

En la Figura 3, el 54,55\% de los estudiantes encuestados consideran que la calidad en su formación profesional es alta; y, el 38,64\% ubica en un nivel muy alto los procesos actuales de formación académica. 


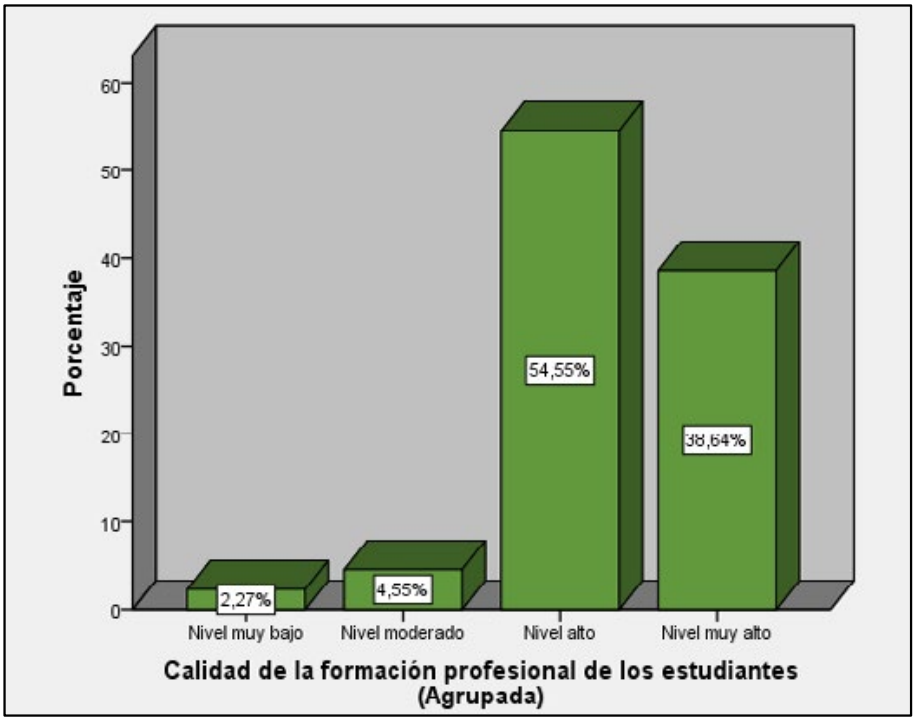

Figura 3. Calidad en la formación profesional de los estudiantes

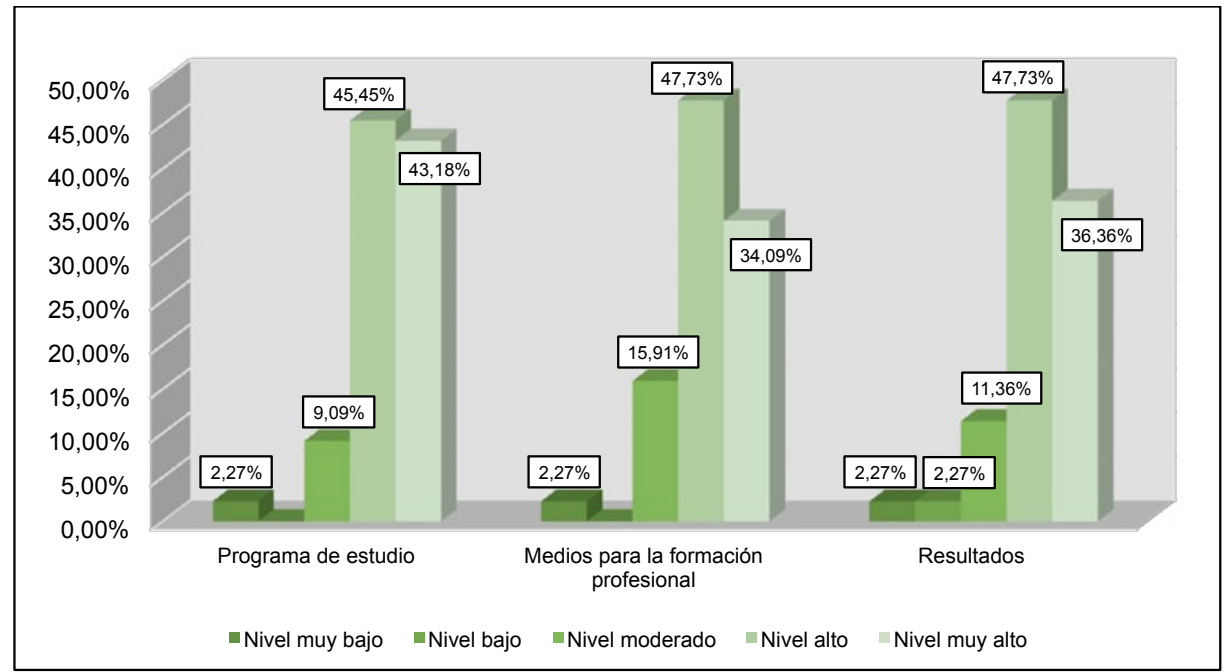

Figura 4. Calidad en la formación profesional de los estudiantes

Analizado esta variable desde sus dimensiones, los estudiantes en una proporción del 45,45\% manifestaron que los programas de estudio tienen un nivel alto para su formación; el 43,18\% los considera con un nivel muy alto, es decir, los alumnos están mayormente conformes respecto a los contenidos, cumplimiento de contenidos, así como son los objetivos del programa de estudio.

Respecto a los medios para la formación profesional, esto en relación a los recursos materiales, técnicos y tecnológicos requeridos y utilizados por docente para impartir la asignatura, así como sus prácticas, los estudiantes en un $47,73 \%$ proporciona una valoración alta, mientras que el 34,09\% opina que se están aplicando medios formativos con estándares muy altos. 
El 47,73\% dio una valoración alta respecto a los resultados o grado de satisfacción con el trabajo realizado por los docentes durante los períodos de clases; el 36,36\% calificó como muy alta los resultados de la gestión docente, haciendo referencia a las actividades, procedimientos y métodos de enseñanza aplicados por el docente que han contribuido con la formación profesional de los estudiantes.

Además de la perspectiva del estudiante, se consideró realizar una autoevaluación del desempeño docente como complemento a la presente investigación a fin de conocer el punto de vista del profesor, es decir, su postura y autocrítica en el desarrollo de la práctica de enseñanza. Los docentes, por medio de la autorreflexión, están en la capacidad de reconocer las fortalezas y limitaciones en su desarrollo profesional. (Aguilar \& Bautista, 2015).

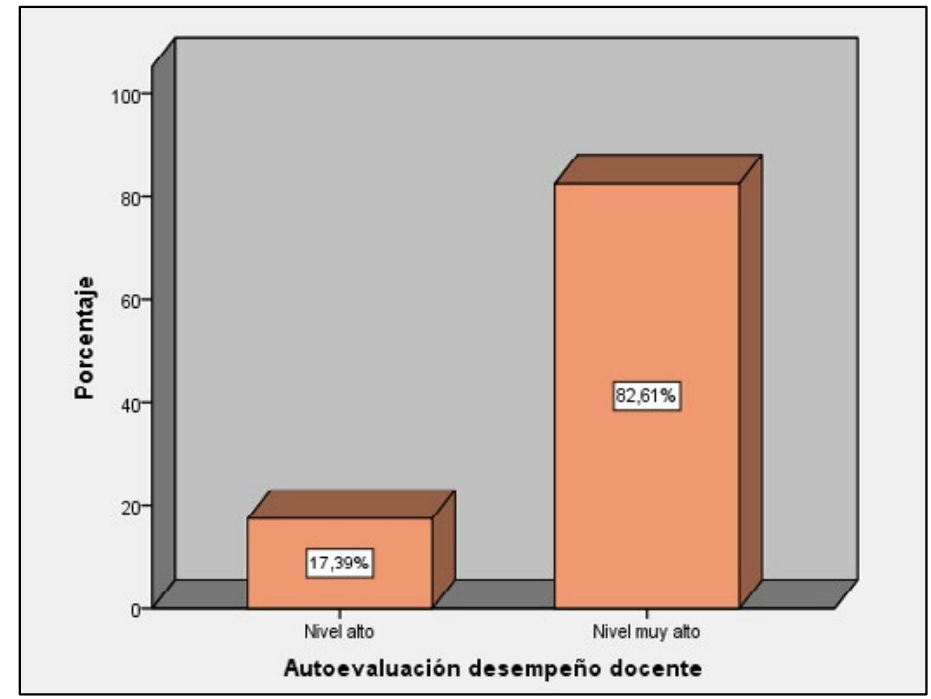

Figura 5. Calidad en la formación profesional de los estudiantes

En la figura 5, se revela que el 82,61\% del profesorado de la Carrera de Bioquímica y Farmacia de la Universidad Técnica de Machala está altamente satisfecho de su labor de enseñanza.

Analizando cada una de las dimensiones evaluadas (ver figura 6), los docentes presentan una valoración alta $(82,61 \%)$ respecto a los procesos de planificación de actividades de estudio. El desarrollo de la enseñanza revela con el 78,28\% un nivel muy alto según la opinión de los docentes. Los procesos de evaluación consideran en un 91,30\% que tienen un nivel muy alto. El $86,96 \%$ respondió que su actitud frente a los estudiantes se ubica en un nivel muy alto. 


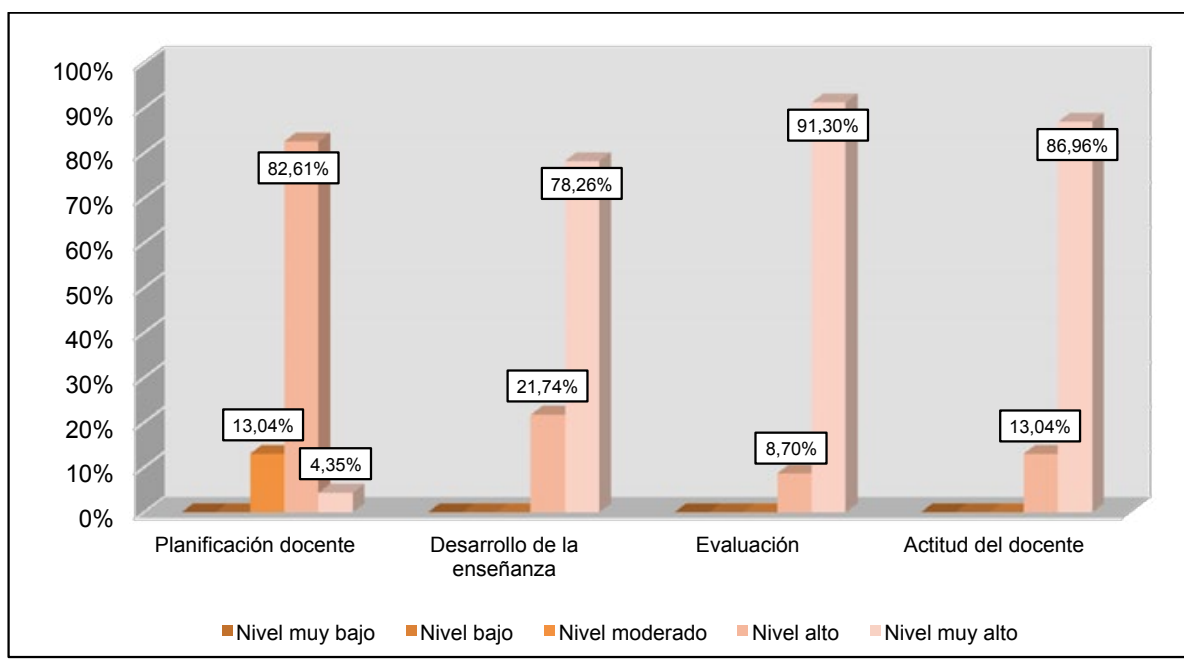

Figura 6. Autoevaluación del desempeño docente por dimensiones

\section{Correlación:}

Para establecer la relación entre las variables contenidas en la hipótesis, se formuló la hipótesis nula y la alternativa, como se indica

- H0 (nula): No existe una incidencia importante entre el desempeño docente y la calidad de la formación profesional de los estudiantes de la Carrera de Bioquímica y Farmacia de la Universidad Técnica de Machala. H1 (alternativa): Si existe una incidencia importante entre el desempeño docente y la calidad de la formación profesional de los estudiantes de la Carrera de Bioquímica y Farmacia de la Universidad Técnica de Machala. El resultado de aplicar el coeficiente de correlación de Spearman, con los datos obtenidos en las encuestas dirigidas a los estudiantes, se muestra en la tabla 3:

\begin{tabular}{|c|c|c|c|c|}
\hline DESEMPEÑO DOCE & & & & \multirow{2}{*}{$\begin{array}{l}\text { CALIDAD EN LA FOR- } \\
\text { MACIÓN PROFESIO- } \\
\text { NAL } \\
, 874^{* *}\end{array}$} \\
\hline \multirow[t]{6}{*}{ Rho de Spearman } & Desempeño docente & $\begin{array}{l}\text { Coeficiente de co- } \\
\text { rrelación }\end{array}$ & 1,000 & \\
\hline & & Sig. (bilateral) & . & 000 \\
\hline & & $\mathrm{N}$ & 44 & 44 \\
\hline & $\begin{array}{l}\text { Calidad en la forma- } \\
\text { ción profesional }\end{array}$ & $\begin{array}{l}\text { Coeficiente de co- } \\
\text { rrelación }\end{array}$ &, $874 * *$ & 1,000 \\
\hline & & Sig. (bilateral) & ,000 & . \\
\hline & & $\mathrm{N}$ & 44 & 44 \\
\hline
\end{tabular}

Tabla 3. Correlaciones

Dado que el valor p-valor (significación bilateral) es menor a 0,05, se rechaza la hipótesis nula, por consiguiente, se demuestra que existe evidencia estadística suficiente para afirmar que existe una incidencia importante entre el desempeño docente y la calidad de la formación profesional de los es-

\section{Cumbres}


tudiantes de la Carrera de Bioquímica y Farmacia de la Universidad Técnica de Machala.

Por otra parte, el coeficiente de Spearman, $\rho$ (rho) $=0,874$; lo cual indica una relación positiva muy fuerte; de acuerdo a la de correlación Hernández, Fernández y Baptista (2014). Por tanto, a mejor desempeño docente universitario se tendrá también una mejor calidad en la formación profesional de los estudiantes.

\section{DISCUSIÓN}

Los resultados positivos obtenidos de la evaluación docente y la incidencia o relación sobre la calidad en la formación de los estudiantes de la Carrera de Bioquímica y Farmacia de la Universidad Técnica de Machala realizada en 2018, son consistentes a los procesos de transformación y mejoramiento del Sistema de Educación Superior que ha venido teniendo desde el año 2008, así lo sostiene Pazmiño (2018) que indica:

Luego de un arduo proceso de regulación, que llevó al cierre de 14 universidades, obligó a las instituciones a que desarrollen renovados esfuerzos, especialmente en el campo de la calidad; permitiendo que recientemente el Ecuador cuenta con Universidades y Escuelas Politécnicas con excelencia académica, cuyo reconocimiento es a nivel nacional e internacional, sin dejar de admitir que aún falta mucho camino por recorrer en ciertas instituciones de Educación Superior para lograr la acreditación y alcanzar los estándares establecidos.

Canalías (2014) además señala que el Estado por primera vez unificó criterios orientados a la calidad de los procesos universitarios, analizados en función de la formación de profesionales, así como priorizando el perfeccionamiento del desempeño de los docentes. En este sentido cabe destacar que Ecuador, durante el 2014 fue uno de los países a nivel mundial con mayor inversión en Educación Superior respecto al tamaño de su economía; 2,1\% del PIB (Abad, López, \& Fernández, 2017). De esta forma, el sistema de educación superior ecuatoriano se enfocó en la constante búsqueda de la excelencia, la pertinencia, producción óptima, transmisión del conocimiento y desarrollo del pensamiento (Véliz, 2018)

Esta situación se debe en gran medida a los procesos de evaluación, que "obligaron a la universidad a plantearse un seguimiento evaluativo en relación al desempeño de sus docentes lo que retorna en el mejoramiento de la calidad de los procesos universitarios" (Gil, Tchinhama, \& Morales, 2017) y, por ende, en la apropiada formación profesional de los estudiantes.

De acuerdo a Márquez y Madueño (2016) la evaluación docente es un factor que favorece tanto la formación del estudiantado como del profesorado, ya que proporciona información para la configuración de estrategias que ayudan en la calidad de la educación que se imparte.

El estudio realizado por López \& Solarte (2013) determinaron que a través del desarrollo de evaluaciones sobre el desempeño pedagógico fue posible mejorar la calidad profesional, los programas y procesos, generando así buenos resultados en la formación de los estudiantes. 
Con los resultados de la presente investigación, se pudo comprobar que el desempeño docente tiene una relación directamente proporcional con los procesos formativos de los estudiantes. De esta manera, el desempeño docente es clave para el desarrollo integral de los estudiantes al incorporar dimensiones afectivas, cognitivas, sociales y morales (Alejandro, Tumbaco, \& Alejandro, 2015); y su constante evaluación permite la valoración, reflexión y control del quehacer del profesor universitario permitiendo la eliminación de debilidades y potenciando sus fortalezas (Sánchez, 2013).

\section{CONCLUSIONES}

De los resultados de la investigación se evidenció que la Universidad Técnica de Machala, en la última década ha realizado grandes esfuerzos en búsqueda de la excelencia y calidad de su gestión académica.

Con la realización de las respectivas evaluaciones en los estudiantes y profesores de la Carrera de Bioquímica y Farmacia, se obtuvieron valoraciones muy positivas, tanto en el desempeño docente como en la calidad en la formación profesional de los estudiantes, lo que refleja el compromiso de la Universidad en el mejoramiento continuo de sus procesos de enseñanza-aprendizaje a través de un cuerpo docente cualificado.

Asimismo, se comprobó que estos dos factores (desempeño docente y calidad en la formación profesional de los estudiantes) están fuertemente relacionados, lo que conlleva a que la evaluación del desempeño docente sea un recurso de aplicación permanente en la Universidad, a fin de controlar y mejorar los aprendizajes obtenidos por los estudiantes.

\section{REFERENCIAS BIBLIOGRÁFICAS}

Abad, G., López, M., \& Fernández, K. (2017). El Sistema de Educación Superior Ecuatoriano visto desde los principios de pertinencia y calidad. Universidad y Sociedad, 46-53.

Aguilar, R., \& Bautista, M. (2015). Perfiles docentes y excelencia: un estudio en la Universidad Técnica Particular de Loja, Ecuador. RIED. Revista Iberoamericana de Educación a Distancia, 225-250. doi:10.5944/ried.18.2.13920.

Alejandro, M., Tumbaco, M., \& Alejandro, C. (2015). El desempeño docente en función de las competencias profesionales en los procesos de acreditación de la Universidad Estatal Península de Santa Elena. Revista Ciencias Pedagógicas e Innovación, 46-51.

Canalías, S. (2014). Evolución histórica de la evaluación en la educación superior ecuatoriana: Pasos hacia la excelencia. Revista Ciencias Pedagógicas e Innovación, 16-25. doi:10.26423/rcpi.v2i2.52

Cuevas, L. (2015). ¿Cómo Evaluar el Desempeño Docente? Investigación y Formación Pedagógica Revista del CIEGC, 24 - 35.

\section{Cumbres}


Dimaté, C., Tapiero, O., González, C., Rodríguez, R., \& Arcila, M. (2017). La evaluación del desempeño docente. Folios, 83-95.

Gil, J., Tchinhama, D., \& Morales, M. (2017). La evaluación del desempeño de los docentes universitarios. Un acercamientoa las realidades educativas. Universidad y Sociedad, 237-241.

Hernández, R., Fernández, C., \& Baptista, M. (2014). Metodología de la investigación. México D.F.: McGraw-Hill.

López, M., \& Solarte, E. (2013). Evaluación por competencias: una alternativa para valorar el desempeño docente universitario. Revista Tendencias, 216-257.

Márquez, L., \& Madueño, M. (2016). Propiedades psicométricas de un instrumento para apoyar el proceso de evaluación del docente universitario. Revista Electrónica de Investigación Educativa, 53-61.

Monereo, C., \& Dominguez, C. (2014). La identidad docente de los profesores universitarios competentes. Educación XX1, 83-104.

Moreira, B. (2015). La práctica docente: Un espacio para el perfeccionamiento de la formación profesional de los estudiantes de la Facultad de Ciencias de la Educación de la Universidad Laica Eloy Alfaro de Manabí. Revista Electrónica Formación y Calidad Educativa, 155-166.

Pazmiño, S. (2018). Análisis del sistema de educación superior en Ecuador desde el 2000 hasta la actualidad "olas, cierre y calidad". Revista de Educación, 45-60.

Ramírez, J. (2017). Modelo teórico de desempeño del docente universitario para la construcción del conocimiento. Revista Scientific, 256-274. doi:10.29394/scientific.issn.2542-2987.2017.0.0.14.256-274

Ronquillo, S., Moreira, C., \& Verdesoto, O. (2016). La evaluación docente una propuesta para el cambio en Facultad de Administración, Universidad ;. Revista Universidad y Sociedad, 125-131.

Sánchez, A. (2013). Modelo para la evaluación del desempeño del docente universitario. Revista La Técnica, 40-49.

Secretaría Nacional de Planificación y Desarrollo. (2013). Plan Nacional de Desarrollo / Plan Nacional para el Buen Vivir 2013-2017. Quito: Senplades.

Véliz, V. (2018). Calidad en la Educación Superior. Caso Ecuador. Atenas, 165180. 
